Original Research Paper

\title{
A Note on the Classification of Compact Homogeneous Locally Conformal Kähler Manifolds
}

\author{
Daniel Guan \\ Department of Mathematics, University of California at Riverside, Riverside, CA 92521 USA
}

Article history

Received: 28-04-2017

Revised: 08-06-2017

Accepted: 10-07-2017

Email: zguan@math.ucr.edu

\begin{abstract}
In this study, we apply a result of H. C. Wang and HanoKobayashi on the classification of compact complex homogeneous manifolds with a compact reductive Lie group to give some more homogeneous space involved proofs of recent classification of compact complex homogeneous locally conformal Kähler manifolds. In particular, we prove that the semisimple part $\mathrm{S}$ of the Lie group action has hypersurface orbits, i.e., it is of cohomogeneity one with respect to the semisimple Lie group $\mathrm{S}$. We also prove that as an one dimensional complex torus bundle, the metrics on the manifold is completely determined by the metrics (which is the same as the Kähler class) on the base complex manifold and the metrics (same as the Kähler class) on the complex one dimensional torus.
\end{abstract}

Keywords: Cohomology, Invariant Structure, Homogeneous Space, Complex Torus Bundles, Hermitian Manifolds, Reductive Lie Group, Compact Manifolds, Ricci Form, Locally Conformal Kähler Manifolds, 1991 Mathematics Subject Classification. 53C15, 57S25, 53C30, 22E99, 15A75

\section{Introduction}

Let $M$ be a complex manifold, $h$ be an Hermitian metrics. If $h$ is locally conformal to Kähler metrics, i.e., for any point $m \in M$ there is an open neighborhood $U$ such that on $U g=e^{f} h$ is a Kähler metrics with a function $f$, we say that $(M, h)$ is a locally conformal Kähler. That is, let $\Omega(\cdot, \cdot)=h(\cdot, J)$, then $\mathrm{d}\left(e^{f} \Omega\right)=0$.

A compact complex homogeneous space with an invariant Hermitian structure was classified by Wang (1954), see also (Hano and Kobayashi, 1960). In fact, they classified the compact complex homogeneous space with compact Lie groups. A Hermitian manifold is a Riemannian manifold. The identity component of the Riemannian isometric group for a compact Riemannian manifold is a compact Lie group. So is the identity component of the Hermitian isometric group for a compact Hermitian manifold.

Therefore, we have:

\section{Lemma 1}

If $M=G / H$ is a compact homogeneous Riemannian manifold with $G$ connected, then $G$ is a subgroup of a compact Lie group. In particular, both $G$ and $H$ are reductive with compact semisimple parts.
We then have (Hano and Kobayashi, 1960 Theorem B):

\section{Lemma 2}

Any compact Hermitian homogeneous manifold is a complex torus bundle over a rational (therefore simply connected) projective homogeneous space.

One could also see (Guan, 1994) page 66, Remark for a detail understanding of this fibration.

Recently, Professor Hasegawa et al proved in (Hasegawa and Kamishima, 2016; Gauduchon et al., 2015):

\section{Proposition 1}

A compact homogeneous locally conformal Kähler manifold $M=G / H$ is a complex 1-dimensional torus bundle over a rational projective homogeneous space.

It is striking that Lemma 2 and Proposition 1 is so close, yet the proofs of Proposition 1 in (Hasegawa and Kamishima, 2016) are so complicated (to us). We have several purposes in this note:

- We shall take a simple approach from Lemma 2 to Proposition 1. As one shall see, our machinery in the compact complex homogeneous spaces theory is powerful enough to solve this problem 
- We shall fill in the details of the argument in (Hasegawa and Kamishima, 2016) (our argument was earlier than (Gauduchon et al., 2015)) from a more complex homogeneous space and higher dimensional aspect

- We shall also take Vaisman's earlier approach into our account

- We shall prove our Main Theorem of the classification of the locally conformal Kähler metrics

\section{Main Theorem}

The manifold is cohomogeneity one under the action of the semisimple part $S$ of the Lie group, i.e., $S$ has hypersurface orbits. $M=N \times S^{1}$ as a homogeneous space (but not necessary as a Riemannian manifold) with $N$ the $S$ orbits. Both the original locally conformal Kähler metrics and the related Kähler metrics are cohomogeneity one under the $S$ action. Moreover, $M$ is a complex one dimensional torus bundle, over a rational homogeneous projective space, which is a finite quotient of a quotient of a positive $C^{*}$ bundle by some action $e^{a}$ with $\operatorname{Re} a \neq 0$. The metrics on $M$, as a submersion, is completely determined by the Kähler class of the base manifold and the Kähler class, as the restriction, of the fiber.

We added some examples to help both experts and the readers understand our arguments.

We obtained some more detail information in our Theorem 2.

We understand that this result is not trivial to the general readers since even a while after the publishing of (Hasegawa and Kamishima, 2016; Gauduchon et al., 2015), we had some discussion of this result and Lemma 6 with Professor Hasegawa. At the beginning of Sept. 2012, Professor Hasegawa visited us and showed us their work on the classification of compact homogeneous locally conformal Kähler manifolds. They seemly had a difficulty to publish. Although not being a referee, as this was an important result, I came up with these proofs in this note. I gave a talk in UCR on Oct. 5, 2012. I told Professor Hasegawa of my proofs. Although my proofs might be simpler and more convincing, they were hesitated to change their original proof. These results were also announced in (Cernea and Guan, 2015). Although Theorem 1 might be kind of trivial on the group level by the Annals paper (Hochchild and Serre, 1953), it was not very clear to us that it is also true for the coset space. Instead, we put some technical details from the structure of rational projective homogeneous space as our Lemma 4 and we also put Lemma 7 in our talk as Theorem 2 . We successfully avoided the Lee field in the second section, which might cause a lot of confusions when the structure of the Hermitian metrics was not known. Also, it is very easy to get confused when people use the left invariant vector fields on the group in the same time with right invariant vector fields on the manifold. Koszul (1955) serves as a good example. It is well-known in the homogeneous space theory. We try to avoid any confusion as much as possible. In the proof of Theorem 2, we extended the proof of Hasegawa et al for the parallel property of $\theta$. To make the proof complete, we have to use a submersion calculation which was well known for the symmetric spaces. Because of the Theorem 1 in the second section, we can now deal with the Lee field freely in the third section. To include the Lee field in the Lie algebra, we use the full isometry group in the third section.

Homogeneous spaces is always a hot and classical topic in Mathematics. We list some good references as (Borel and Remmert, 1962; Chevalley, 1968; Cernea and Guan, 2015; Dorfmeister and Guan, 1991; Dorfmeister and Nakajima, 1988; Guan, 2002; 1996; Gauduchon et al., 2015; Hasegawa and Kamishima, 2016; Hano and Kobayashi, 1960; Hochchild and Serre, 1953; Koszul, 1955; Mostow, 1961; Matsushima, 1957; Nakamura, 1975; Nomizu, 1954; Tits, 1971; Wang, 1954) as examples at the end. From our Theorem 1, we also obtained that the semisimple part $S$ of the Lie group acts on $M$ with cohomogeneity one action, i.e., with hypersurface orbits. The reduction of $M$ by $S$ is $S^{1}$. That is $M=N \times S^{1}$ as a homogeneous space and the related Kähler metrics are cohomogeneity one under the $S$ action. We also are able to classify the cohomogeneity one case. We shall deal with it in a different paper. Our early purpose for this note is giving an alternative explanation of (Hasegawa and Kamishima, 2016). However, after receiving some concerns, we decided to add the Corollary 1 and therefore, the Main Theorem to make our Theorem 1 a little bit more different from the contents in (Hasegawa and Kamishima, 2016). We also added some examples from the original Hopf surfaces (Kobayashi and Nomizu, 1996, page 137).

Let us give a quick description for the case when $\operatorname{dim}_{C}$ $M=2$. According to Lemma 2 above, $M$ is either a complex torus of complex dimension 2 or a complex one dimensional torus bundle over $C P^{1}$. The simply connected case was excluded by the locally conformal Kähler (but nonkähler) condition. For a complex torus, all the homogeneous hermitian metrics are actually Kähler. Therefore, we obtain the Proposition 1 . We also notice that the manifold $M$ might not be a Hopf surface itself but a finite covering of it. However, any homogeneous hermitian metrics comes from the Hopf surface covering and therefore, all the discussion about the Hopf surfaces in the later sections in this note apply to $M$.

Also, in this case, the semisimple part of the group comes down to be an effective isometric subgroup on $C P^{1}$ and therefore is $S U(2)$. This is because that the isometric group on the torus fiber is abelian. Now, the dimension of $S U(2)$ is 3 and the action is cohomogeneity 
one, i.e., it has hypersurfaces as orbits. To be homogeneous for $M=G / H$, the center of $G$ is at least of a real dimension 1 and at most of real dimension 2. $G$ is locally either $S U(2) \times R$ or $S U(2) \times C$. Moreover, the $S U(2)$ orbits are $S^{1}$ bundles over $C P^{1}$. That leads to a line bundle and therefore a $C^{*}$ bundle $M^{*}$ over $C P^{1}$.

$M^{*}$ is a covering of $M$. Let $M_{a}=\left(C^{2}-\{0\}\right) /(a),(a)=$ $\left\{\left.a^{i}\right|_{i \in Z}\right\}$ with $a \in C^{*}$ and $|a| \neq 1$, be a Hopf surface, then $M_{a}^{*}=C^{2}-\{0\}$. Then $M_{a}^{*}$ is a finite covering of $M^{*}$. We denote the earlier one simply by $C^{2,{ }^{*}}$. The covering map $C^{2, *}$ to $M^{*}$ introduces a Hopf surface covering over $M$ (introduced by the corresponding $S^{1}$ bundles, which introduces a finite torus covering for each fiber).

This is our Main Theorem (and Corollary 1) for the complex dimension 2 case.

\section{First Proof of Proposition 1, from Lemma 2}

In the Introduction we noticed that on $U d\left(e^{f} \Omega\right)=0$. That is:

$$
e^{f}(d f \wedge \Omega+d \Omega)=0
$$

We define $\theta=-d f$. Then $d \Omega=\theta \wedge \Omega$. Since $\Omega$ is nondegenerate, $\theta$ is uniquely determined if $\mathrm{n}=\operatorname{dim}_{C} M>$ 1 . In the following, we always assume that $n \neq 1$ since when $n=1, \mathrm{M}$ is always Kähler.

\section{Lemma 3}

$\theta$ is closed, represents a nonzero cohomology class if $h$ is not conformally Kähler itself. In the compact homogeneous case, $\theta$ is in the dual of the center.

\section{Proof}

$d \theta=d(-d f)=0$ implies that $\theta$ is closed. If $\theta$ represents a zero class in the cohomology, then $\theta=d F$ for a global function $F$. That is, $e^{-F} h$ is Kähler. Since $G$ is reductive, the Lie algebra of $G$ is $s+t$ with $\mathrm{s}$ compact semisimple and t abelian. Since $\Omega$ and $d \Omega$ are invariant under $G$, so is $\theta$. The dual of the Lie algebra is $s^{*}+t^{*}$. The pull back of $\theta$ to $G$ is also closed. Now we use $\alpha$ as the left invariant 1 -form on $G$ such that $\alpha$ is the same as $\theta$ at the identity point. Then $\alpha$ has the same property as $\theta$, i.e., closed and $d \beta=\alpha \wedge \beta(\bmod \mathrm{H})$ with $\beta$ the left invariant 2 -form corresponding to the pullback of $\Omega$ (they are equal at the identity). $\alpha=\alpha_{s}+$ $\alpha_{t} \in s^{*}+t^{*}$, where $\alpha_{s} \in s^{*}$ and it is not closed unless it is zero while $\alpha_{t} \in t^{*}$ is always closed. Therefore, $\alpha_{s}$ is also closed and hence is zero. We have that $\alpha$ is in $t^{*}$. Since $t$ is the center, we can identify $\alpha$ to be the pullback of $\theta$ and still denote it as $\theta$.

Next result is one of the major parts of the proof. We need prove.

\section{Theorem 1}

$\Omega=k(\theta \wedge J \theta-d(J \theta))$ and the torus bundle is a submersion.

\section{Corollary 1}

The manifold is cohomogeneity one under the action of the semisimple part $S$ of the Lie group, i.e., it has hypersurface orbits. $M=N \times S^{1}$ as a homogeneous space (but not necessary as a Riemannian manifold) with $N$ the $S$ orbits. The related Kähler metrics are cohomogeneity one under the $S$ action. Moreover, $M$ is a complex torus bundle over $G / K$ and a finite quotient of a quotient of a positive $C^{*}$ bundle by some action $e^{a}$ with $\operatorname{Re} a \neq 0$. The Lie group action can be locally isomorphic to $S \times R$ or $S \times$ $C^{*}$. In the first case, the Lee field may and may not be in the Lie algebra but in the second case the Lee field is in $C^{*}$. The metrics on $M$ is completely determined by the metrics (actually the Kähler class) on the rational projective space and the metrics (same as the Kähler class) on any one of the complex one dimensional torus fiber.

Notice that this Theorem is basically from (Hasegawa and Kamishima, 2016) by using (Hochchild and Serre, 1953). However, the result from (Hochchild and Serre, 1953) only stated for the Lie group level. That is the reason that we need give a detail account of this result as it is crucial to all the proofs.

Before we prove our Theorem 1, we need a structure of the Lie algebra $\mathrm{G}$ of $G$, from e.g., (Wang, 1954; Hano and Kobayashi, 1960; Guan, 2002). Let $H$ be the Lie algebra of $H$. We have a tower of two fiber bundles:

$$
G \rightarrow G / H \rightarrow G / K
$$

where, $G / K=S / K_{S}$ is the rational projective homogeneous space and $K / H$ a complex torus. $K_{S}$ is a centralizer of a torus in $S . \mathrm{G}=s+t=\mathrm{H}+m=m_{0}+m_{1}$ with $m_{0}$ the tangent space of the rational projective space and $m_{1}$ the tangent space of the complex torus. Let $\mathrm{K}$ and $\mathrm{K}_{s}$ be the Lie algebra of $K$ and $K_{S}$ respectively, then $t \subset \mathrm{K}, m_{0} \subset s$. Let $H_{S}=H \cap S$, then the semisimple part of $H_{S}$ is the semisimple parts of both $K_{S}$ and $H$. Let $\mathrm{H}_{s}$ be the Lie algebra of $H_{S}$.

\section{Lemma 4}

For any given $\gamma \in m_{0}^{*}$, we have $d \gamma \neq 0$. For any $\gamma \in m_{1}^{*} d \gamma \neq 0$ unless $\gamma \in t^{*}$. For any $\gamma \in m^{*} d \gamma \in 0$ unless $\gamma \in t^{*}$.

\section{Proof}

Of course, the first and the second statements are actually special cases of the third statement. The first one looks stronger but one might notice that the intersection of $m_{0}^{*}$ and $t^{*}$ is trivial. 
I am putting all three statements in the Lemma just for the convenience of the readers. One could check the first statement easily by the well-known properties of the rational projective space, e.g., in (Hano and Kobayashi, 1960; Guan, 2002; Tits, 1971). Or we just notice that $G / K$ is simply connected. Similarly for the second statement that $d m^{*} \subset{ }_{1} \subset \wedge^{2} m^{*}{ }_{0}(\operatorname{modH})$ by $m_{1}$ being in the center of $K$. To get the third statement, one only need to compare the term types appeared in $d \gamma$ from the first and the second statement. We leave the details to the readers. One could also use a spectral sequence argument as in (Guan, 1996) that the only real contribution for $H^{1}(G / H, \mathbf{R})$ comes from $m_{1}^{*}$ since $H^{1}(G / K, \mathbf{R})=0$.

If all those arguments above do not satisfy the reader, just take the same reason as for why $\theta$ is in the center of the Lie algebra. The point is that if $d \gamma=0$, then:

$$
0=d \gamma(x, y)=\gamma([x, y])
$$

That is, the Lie algebra of $S$ is in the kernel of $\gamma$. Now, we are ready to prove the Theorem 1 :

\section{Proof of Theorem 1}

By diagonalizing the Hermitian form we can assume:

$$
\Omega=k \theta \wedge J \theta+\Omega_{1}
$$

with $\Omega_{1}$ perpendicular to $\theta$ and $J \theta$. Then:

$$
d \Omega=-k \theta \wedge d(J \theta)+d \Omega_{1}
$$

This implies, $d \Omega_{1}=0$. By Lemma 4, we have:

$$
\Omega_{1}=d \alpha+\Omega_{0}(\bmod \mathrm{H})
$$

with $\Omega_{0} \in \wedge^{2} t^{*}$ and $\alpha \in s^{*} . \Omega_{0}$ is perpendicular to $\theta$ in the Lie algebra. If $t$ is an center element corresponding to $\theta$, then $\Omega_{0}(t)=0$ :

$$
\begin{aligned}
& \Omega=k \theta \wedge J \theta+d \alpha+\Omega_{0}(\bmod \mathrm{H}) \\
& d \Omega=-k \theta \wedge d(J \theta)=\theta \wedge \Omega=\theta \wedge\left(d \alpha+\Omega_{0}\right)(\bmod \mathrm{H})
\end{aligned}
$$

We have $d(J \theta) \in \wedge^{2} s^{*}$ implies that $\Omega_{0}=0$ and:

$$
-k d(J \theta)=d \alpha(\bmod \mathrm{H})
$$

Therefore, we get the Theorem 1.

To get Proposition 1, we just notice that $-k d(J \theta)$ is the Kähler form of the rational projective homogeneous space $M_{0}$ which contains the semisimple part of $K_{S}$ in its isotropic subgroup and hence $M_{0}$ is $G / K$. The rank of - $k d(J \theta)$ is obviously $n-1$. Therefore, $G / H$ is a 1 dimensional complex torus bundle over $G / K$. The map $G / H$ over $G / K$ is a Hermitian submersion.

\section{Relation to Vaisman's Method}

Theorem 1 might be a little bit too abstract to the readers, let us look at the standard Hopf surface:

$$
\left(C^{2}-\{(0,0)\}\right) /\left(e^{a}\right)
$$

with $\operatorname{Re} a \neq 0$. There are metrics $k|z|^{-2} d z \wedge d \bar{z}$ :

$$
f=\ln |z|^{2}, \theta=-d f=-d\left(|z|^{2}\right) /|z|^{2}
$$

Actually, any cohomogeneity one Kähler metrics of a principal $C^{*}$ bundle over a rational projective homogeneous manifold is fully understood. One might see (Guan, 1995). We shall discuss the Hopf surfaces a little bit more after Lemma 6 .

From our Theorem 1, we could see that the Lee field is in the center of the Lie algebra of the isometric group. There was a concern of the described Lee field, with an example in the proof of the Proposition 3 in (Hasegawa and Kamishima, 2016). But it seems to us that there is no problem with above Hopf surface with any given complex number $a$ above and it fits with this metric. That is, the Lee field has nothing to do with $a$ and is in the center. However, we do see that the geodesics perpendicular to the $S$ orbits might not be closed.

Earlier, Vaisman proved that Proposition 1 is true if $\theta$ is parallel.

\section{Lemma 5}

If $M$ is a compact homogeneous locally conformal Kähler manifold, $\theta$ is harmonic. That is, $d \theta=0=\delta \theta$.

Proof

Since $M$ is homogeneous, $\delta \theta$ is a constant:

$$
\delta \cdot \text { volume }=(\delta \theta, 1)=(\theta, d(1))=0
$$

In (Vaisman, 1982) Theorem 2.1, Vaisman proved the following Proposition:

\section{Proposition 2}

Let $(M, h)$ be a compact locally conformal Kähler manifold and assume that its underlying structure consists of related Kähler metrics with nonnegative Ricci curvatures. Then there is a global function $\varphi>0$ on $M$ such that $(M, \varphi h)$ is a generalized Hopf manifold.

A locally conformal Kähler manifold is a generalized Hopf manifold if $\nabla \theta=0$, i.e., $\theta$ is 
parallel. See Vaisman (1982) page 232. He basically found a $\varphi$ such that $\theta$ for $\varphi h$ is harmonic, which we already achieved in Lemma 5 . Then he proved that with the Ricci curvature condition, harmonic implies that $\theta$ is parallel. See his proof from page 234 (the second paragraph) to 235 in (Vaisman, 1982).

\section{Lemma 6}

If $M$ is a compact homogeneous locally conformal Kähler manifold, then:

$$
\Omega_{l}=k \theta \wedge J \theta-l d(J \theta)
$$

is also a locally conformal Kähler structure.

\section{Proof}

By a direct calculation.

We notice that the new metrics in the Lemma 6 is different from that in Theorem 1 since we have a different $\theta$ in this new metrics. Comparing this to Theorem 1, if we let $\theta^{\prime}=m \theta$ be the new Lee form, then $k^{\prime} m^{2}=k$ and $k^{\prime} m=l$. This implies $m=k / l k^{\prime}=$ $l^{2} / k$. In the case of Hopf surfaces, the change in the second term (the term with $l$ ) comes from the pullback of the standard metrics on:

$$
C P^{1}=\left(C^{2}-\{(0,0,)\}\right) / C^{*}
$$

which is:

$$
i \partial \bar{\partial} \log |z|^{2}=\frac{i}{|z|^{4}} w \wedge \bar{w}
$$

with $w=z_{2} d z_{1}-z_{1} d z_{2}$.

By Corollary 1, all the homogeneous locally conformal Kähler metrics on the Hopf surfaces have above form.

In general, Theorem 1 gives a $C^{*}$ (or line) bundle over $M_{0}=G / K$ such that $M$ is the quotient of the $C^{*}$ bundle by some $e^{a}$ with $\operatorname{Re} a \neq 0$. $J \theta$ is more like the connection of the circle bundle and $-d J \theta$ is more like the Chern class of line bundle (or the Euler class of the circle bundle). See Kobayashi (1987) page 10 to 12 for the line bundle calculations.

The following Theorem implies the main results in both papers of Hasegawa and Kamishima, 2016 and Gauduchon et al., 2015, i.e., $\theta$ is parallel (or $M$ is Vaisman). However, it also gives some extra information on the Ricci curvature of the related Kähler metrics when $l$ is big. That relates our argument to Proposition 2 and is completely new, at least at the end of 2012 when we gave a talk in University of California at Riverside.

\section{Theorem 2}

When $l$ is big enough, the new Hermitian structure is locally conformal to Kähler metrics with nonnegative Ricci curvatures. Therefore, $\theta$ is parallel.

\section{Proof}

Let us just calculate the Ricci curvature of the related Kähler metric of $\Omega_{l}$. We notice that $d \Omega_{l}=-k \theta \wedge d(J \theta)=$ $(k / l) \theta \wedge \Omega_{l}$ and therefore, $f_{l}=(k / l) f$. Ricci curvature of the Kähler metric is:

$$
\begin{aligned}
& \partial \bar{\partial} \log \left(e^{n(k / l)} \Omega^{n}\right)=n(k / 2 l) d(J \theta) \\
& +\operatorname{Ricci}(G / K)=n(k / 2 l) d(J \theta)+k_{0} d(J \theta)
\end{aligned}
$$

if the Ricci curvature of $G / K$ is $k_{0} d(J \theta)$. Actually, the relation between $d(J \theta)$ and the Ricci form of $G / K$ does not matter since $d(J \theta)$ is a pullback closed 2-form from $G / K$, which is Kähler-Einstein. Another way to look at this is that even if the Ricci curvature of $G / K$ is not $k_{0} d(J \theta)$ but $d \gamma$ with some $\gamma$ from the center of $K$ (Guan, 1994, page 66 , Remark for a detail understanding of this calculation), we still have:

$$
-C d \gamma<d(J \theta)<C d \gamma
$$

for some constant $C$. For more details of the structure of $H^{1,1}(G / K)=H^{2}(G / K, \mathbf{R})$ (been a Fano manifold) see, for example, (Dorfmeister and Guan, 1991). We notice that $G / K$ is Kähler-Einstein (Guan, 2002) and the Ricci form fibration is exactly the torus fibration. Therefore, when $l$ is big, the Ricci curvature of the related Kähler metrics is close to the pullback Ricci curvature from $G / K$ and is nonnegative. This finished the first part of Theorem 2 .

For the second part, we could follow Vaisman's argument in the proof of Proposition 2 to prove $\nabla^{l} \theta=0$ when $l$ is big enough. Then:

$$
\nabla \theta=\nabla^{k} \theta=0
$$

follows from that as a rational function of $l, \nabla^{l} \theta$ is zero on an open interval.

However, here I would like to use an argument similar to Hasegawa et al. To prove that $\theta$ is parallel, one might prove $\psi=h^{*}(, \theta)$ is parallel.

The reason is following:

$$
\begin{aligned}
& \nabla(\theta(X))=(\nabla \theta)(X)+\theta(\nabla X) \\
& =\nabla(h(\psi, X))=h(\nabla \psi, X)+\theta(\nabla X)
\end{aligned}
$$

hence $(\nabla \theta)(X)=h(\nabla \psi, X)$. That is, $\nabla \psi=0$ implies $\nabla \theta=0$. 
Before we go further, we notice that the Lee field $\psi$ might not be in the Lie algebra. To include the Lee field in the Lie algebra, we now let $G$ be the identity component of the full isometric group. From Theorem 1, we notice that $M$ is the quotient of a $C^{*}$ bundle over $S / K_{S}$. $J \theta$ acts on $C^{*}$ as the circle action and $\theta, \psi$ corresponds to the expanding direction. Therefore, $\psi$ is commute with $S$ and hence is in the center.

Now, we pull $\psi$ back to $G$, since it is in the center, it is both right and left invariant. According to (O'Neill, 1983) page 311, part (2) of the Proposition 11.22, there is one and only one $G$ left-invariant and $\operatorname{Ad}(H)$ invariant metrics on $G$ such that $G \rightarrow G / H$ induces a Riemannian submersion to $h$. We again denote it by $h$. We want to apply (O'Neill, 1983) page 212, part (3) of Lemma 7.45 to our situation. That is, if $\psi$ is parallel on $G$, so is it on $G / H$.

Now, let $x, y$ be two left invariant vector fields on $G$ (nonnecessary the pullback ones which are only rightinvariant), then:

$$
0=x(h(\psi, y))=h\left(\nabla_{x} \psi, y\right)+h\left(\psi, \nabla_{x} y\right)
$$

and:

$$
0=y(h(\psi, y))=h\left(\nabla_{y} \psi, x\right)+h\left(\psi, \nabla_{y} x\right)
$$

since $0=[\psi, x]=\nabla_{\psi} x-\nabla_{x} \psi$. Substracting the first two identities, we get:

$$
0=h\left(\nabla_{x} \psi, y\right)-h\left(\nabla_{y} \psi, x\right)
$$

Comparing with the third identity, we have $h\left(\nabla_{x} \psi, y\right)$ $=0$. That is, $\nabla \psi=0$ on $G$. By Lemma 7.45 in (O'Neill, 1983), we get that $\nabla \psi=0$ on $G / H$ also. Therefore, $\nabla \theta=$ 0 as desired.

\section{Conclusion}

Therefore, we proved in this note that any compact complex homogeneous space which is a one dimensional complex torus bundle over a rational projective homogeneous space $Q$ and is a quotient of a homogeneous positive $C^{*}$ over $Q$, has homogeneous locally conformal Kähler structures described in the Lemma 6. On the other hand, all the compact complex homogeneous space with a homogeneous locally Kähler structure must be one of them.

\section{Acknowledgment}

Here, I would like to take this opportunity to express my appreciation to Professor Hasegawa for sharing their most recent work with me, for discussions and sending me Vaisman's (1984). I also like to dedicate this paper to Professor Kobayashi. I also thank Professor Dorfmeister for showing me (Wang, 1954; Hano and Kobayashi, 1960) when I was in the University of Georgia. That was one of reasons after he left the University of Georgia I eventually moved to Berkeley and followed Professor Kobayashi.

\section{Ethics}

This paper serves as a different proof of the classification of compact complex homogeous locally conformal Kähler manifolds obtained by Hasegawa et al. and this proof was given in 2012. We gave a talk on this in 2012. It was also announced in Cernea et al. (2015).

\section{References}

Borel, A. and R. Remmert, 1962. Über kompakte homogene Kählersche Mannigfaltigkeiten. Math. Ann., 145: 429-439.

Chevalley, C., 1968. Théorie Des Groupes de Lie. Groupes Algebriques, Theoremes Generaux Sur Les Algebres De Lie, Hermann.

Cernea, P. and D. Guan, 2015. Killing fields generated by multiple solutions to the Fischer-Marsden equation. Intern. J. Math., 26: 1540006-1540006.

Dorfmeister, J. and Z. Guan, 1991. Fine structure of reductive pseudo-Kählerian spaces. Geom. Dedi. 39: 321-338. DOI: 10.1007/BF00150759

Dorfmeister, J. and K. Nakajima, 1988. The fundamental conjecture for homogeneous Kähler manifolds. Acta Math., 161: 23-70.

DOI: $10.1007 / \mathrm{BF} 02392294$

Guan, D., 2002. Classification of compact complex homogeneous spaces with invariant volumes. Trans. Am. Math. Soc., 354: 4493-4504.

Guan, D., 1996. A splitting theorem for compact complex homogeneous spaces with a symplectic structure. Geom. Dedi. 63: 217-225. DOI: $10.1007 / \mathrm{BF} 00148221$

Guan, D., 1994. Examples of compact holomorphic symplectic manifolds which admit no Kähler structure. Geometry and Analysis on Complex Manifolds (Professor S. Kobayashi's 60-th birthday) World Scientific Publishing Co.

Guan, D., 1995. Existence of extremal metrics on compact almost homogeneous Kähler manifolds with two ends. Trans. Am. Math. Soc., 347: 2255-2262. DOI: $10.2307 / 2154938$

Gauduchon, P., A. Moroianu and L. Ornea, 2015. Compact homogeneous locally conformal Kähler manifolds are Vaisman. Math. Ann., 361: 1043-1048.

Hasegawa, K. and Y. Kamishima, 2016. Compact homogeneous locally conformally Kähler manifolds. Osaka J. Math., 53: 683-703. 
Hano, J. and S. Kobayashi, 1960. A fibering of a class of homogeneous complex manifolds. Trans. Am. Math. Soc., 94: 233-243.

Hochchild, G. and J.P. Serre, 1953. Cohomology of lie algebras. Annals Math., 57: 591-603. DOI: $10.2307 / 1969740$

Kobayashi, S., 1987. Differential Geometry of Complex Vector Bundles. 2nd Edn., Iwanami Shoten, Tokyo, ISBN-10: 069108467X, pp: 304.

Kobayashi, S. and K. Nomizu, 1996. Foundations of Differential Geometry. 1st Edn., John Wiley and Sons, New York, ISBN-10: 0471157333, pp: 344.

Koszul, J.L., 1955. Sur la forme hermitienne canonique des espaces homogènes complexes. Canad. J. Math., 7: 562-576. DOI: 10.4153/CJM-1955-061-3

Mostow, G.D., 1961. Cohomology of topological groups and solvmani-folds. Ann. Math., 73: 20-48.

Matsushima, Y., 1957. Sur les espaces homogènes kählériens d'un groupe de lie réductif. Nagoya Math. J., 11: 53-60.

DOI: $10.1017 / \mathrm{S} 0027763000001938$
Nakamura, I., 1975. Complex parallelizable manifolds and their small deformations. J. Diff. Geom., 10: 85-112.

Nomizu, K., 1954. On the cohomology of compact homogeneous spaces of nilpotent lie groups. Ann. Math., 59: 531-538. DOI: 10.2307/1969716

O'Neill, H., 1983. Semi-Riemannian Geometry with Applications to Relativity. 1st Edn., Academic Press, New York, ISBN-10: 0080570577, pp: 468.

Tits, J., 1971. Représentations linéaires irréductibles d'un groupe réductif sur un corps quelconque. J. Reine Angew. Math., 247: 196-220.

Vaisman, I., 1982. Generalized Hopf Manifolds. Geom. Dedicata, 13: 231-255. DOI: 10.1007/BF00148231

Vaisman, I., 1984. A servey of generalized hopf manifolds. Rend. Sem. Mat. Univ. Politecn. Torino.

Wang, H.C., 1954. Closed manifolds with homogeneous complex structure. Am. J. Math., 76: 1-32.

DOI: $10.2307 / 2372397$ 